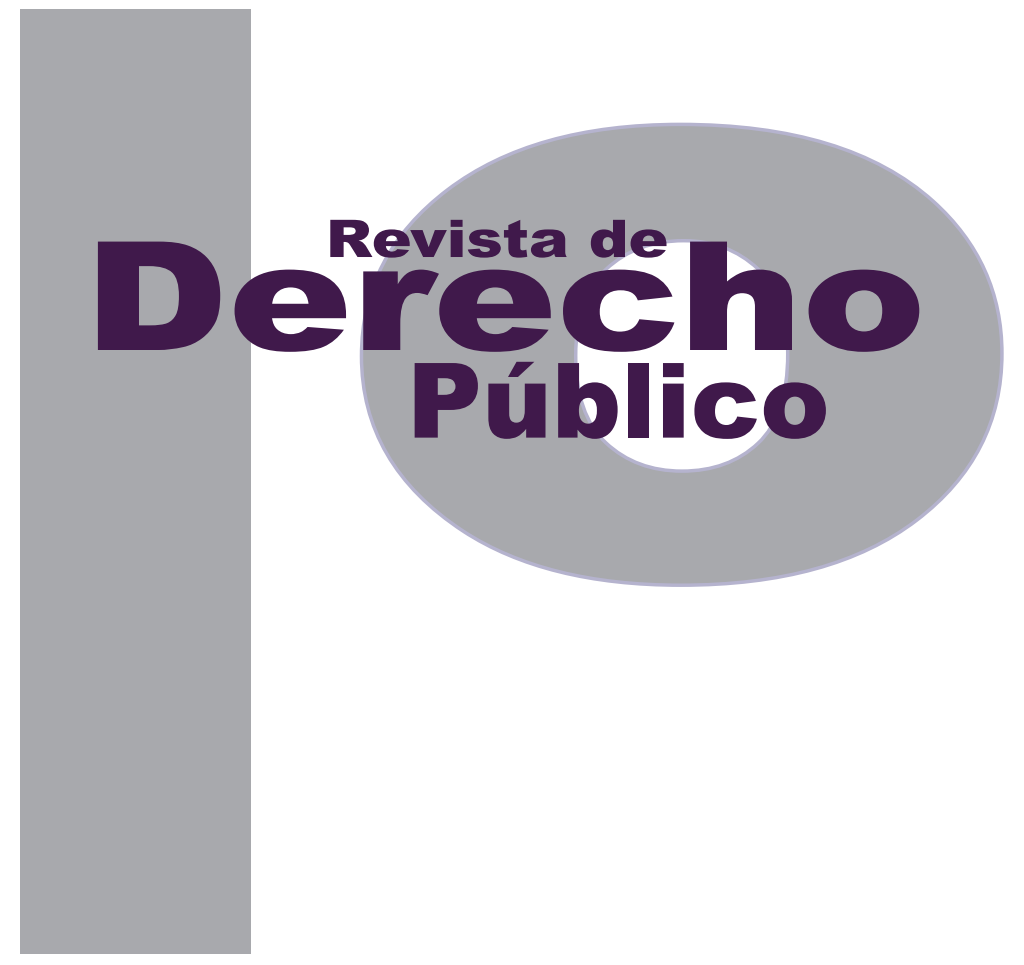

\title{
La Agencia Nacional de Hidrocarburos A LA LUZ DE LA NOCIÓN DE AGENCIA EN EL DERECHO COMPARADO
}

JaIme ANdrés Melo Marulanda

Universidad de los Andes

Facultad de Derecho

Revista de Derecho Público N. ${ }^{\circ} 30$

Enero - Junio de 2013. ISSN 1909-7778 


\title{
La Agencia Nacional de Hidrocarburos a la luz de la noción de agencia en el derecho comparado
}

\author{
Jaime Andrés Melo Marulanda*
}

\section{RESUMEN}

Este artículo tiene como fin exponer, desde un análisis del derecho comparado, las distintas maneras en las que se puede regular la explotación de hidrocarburos. Básicamente la regulación depende del sistema jurídico que se esté estudiando. Es usual que en el common law se utilice el concepto de agencia como un órgano encargado de establecer la forma en la que se regulan distintos sectores de la economía. En Colombia existe una regulación similar a la establecida en Francia, sin embargo, la influencia que Estados Unidos ha tenido en las últimas décadas alrededor del mundo, no solo ha sido a nivel militar, político, social o económico, sino también a nivel institucional. Muchas instituciones del sistema anglosajón se han incorporado en distintos ordenamientos jurídicos. La agencia es una institución que se ha venido incorporando en distintos ordenamientos jurídicos

\section{ABStRACT}

This article aims to explain, from a comparative law analysis, the different ways in which you can regulate the exploitation of hydrocarbons. Basically the regulation depends on the legal system being studied, it is usual that the common law concept of using the Agency as a body to establish how regulating different sectors of the economy. In Colombia there is a regulation similar to that established in France, however, the influence that the U.S. has had in the last decades, around the world, has not only been at the military, political, social or economic, but also at the institutional level. Many institutions of common law have been incorporated in different jurisdictions. The Agency is an institution that has been incorporated in different jurisdictions with different legal traditions to its origin. Therefore it is interesting to conduct a study of the agency purchased in different jurisdictions, in order

Estudiante de Derecho de la Universidad de los Andes, con opción en Economía. Realizó intercambio académico en la Universidad Carlos III de Madrid, en el año 2011. Curso de inglés jurídico en Kings Colles (Londres) en el 2012. Trabaja en la apertura de procesos y en resolución de recursos de reposición en la Superintendencia de Puertos y Transporte. Es miembro de Programa PAllS en la Universidad de los Andes. 
que tienen una tradición legislativa distinta a la de su origen. Por lo anterior, es interesante realizar un estudio comparado de esta institución en distintos ordenamientos jurídicos, con el fin de establecer si existe alguna similitud entre los diversos tipos de agencia que hay en el mundo y la Agencia Nacional de Hidrocarburos.

Palabras clave: agencia, hidrocarburos, derecho comparado, autoridades administrativas independientes, entes descentralizados, entes reguladores, economía de mercado, utilidades, órgano ejecutivo, órgano legislativo. to establish whether there is any similarity between the different types of agency that is in the world and the National Hydrocarbons Agency.

KEY wORDS: Agency, hydrocarbons, comparative law, independent administrative authorities, decentralized entities, regulators, market economy, profits, executive, legislature. 


\section{SUMARIO}

Introducción - I. ¿CON QUÉ FIN SE REGULA? - II. LA AGENCIA EN LOS ESTADOS UNIDOS DE AMÉRICA - A. Historia - B. Marco jurídico - C. Tipo de funciones que puede tener una agencia - D. Las agencias pueden ser de dos tipos: agencias independientes y las agencias que dependen del Ejecutivo - E. Regulación en la producción de hidrocarburos - III. LA AGENCIA EN CANADÁ - A. En Canadá se encuentran las agencias que dependen del Ejecutivo y las independientes - B. Regulación en materia energética e hidrocarburos - IV. LA AGENCIA EN AUSTRALIA - V. LA AGENCIA EN FRANCIA - A. La evolución de la autonomía en los entes estatales en Francia - VI. LA AGENCIA EN ESPAÑA - A. La elección de los directivos en las Autoridades Administrativas Independientes AAI - VII. LA AGENCIA EN SUECIA - VIII. LA AGENCIA EN EL REINO UNIDO - A. Regulación en la explotación de hidrocarburos - IX. LA AGENCIA EN COLOMBIA: ORIGEN Y DESARROLLO - A. Los tipos de contratos de hidrocarburos como forma de regular la producción de este recurso no renovable $-B$. Historia de la regulación de hidrocarburos $-C$. El concepto de agencia es nuevo en Colombia - X. CONCLUSIÓN - Bibliografía. 
Introducción

En Colombia, hasta el año 2003, el ente encargado de la regulación de la exploración y explotación de hidrocarburos fue Ecopetrol (Empresa Colombiana de Petróleos). Esta función le fue suprimida por el Estado porque había cierto nivel de desconfianza por parte de los inversionistas, en cuanto a la imparcialidad que podría tener una empresa petrolera que se encargaba de competir y al mismo tiempo de regular la actividad de hidrocarburos. Por lo anterior, en el año 2003, mediante el Decreto 1760 se creó la Agencia Nacional de Hidrocarburos (en adelante ANH), con la finalidad de separar de Ecopetrol la función reguladora y que esta se pudiera dedicar únicamente a explorar, producir, transportar, refinar y comercializar hidrocarburos y a competir en las mismas condiciones con las demás empresas petroleras. Sin embargo, el solo hecho de crear una agencia no garantiza que esta sea independiente y autónoma en sus decisiones. Lo anterior es relevante en el sentido de que las decisiones regulatorias se basen en criterios técnicos. ¿Qué pasaría si un inversionista extranjero se da cuenta que la agencia encargada de regular la industria en la que él piensa invertir, no toma sus decisiones basándose en concepto técnicos sino, por el contrario, en criterios políticos o, peor aún, influida fuertemente por el poder Ejecutivo? Probablemente lo pensaría dos veces antes de invertir, puesto que podría correr el riesgo que por medio de una regulación administrativa de la agencia, esta termine limitando sus derechos como inversionista.
La noción de agencia fue importada al ordenamiento jurídico colombiano desde el sistema jurídico norteamericano. Por tanto, según este último, debería basarse en dos características: independencia y autonomía. El propósito de este artículo académico es analizar, con base en el derecho comparado, el origen y desarrollo que ha tenido el concepto de agencia en Estados Unidos y su incorporación en varios sistemas jurídicos en el mundo. Todo esto para contestar a la siguiente pregunta de investigación: ¿es la ANH una entidad autónoma e independiente?

Para dar cuenta de esto, el artículo se divide en las siguientes partes: la primera, que se encargará de hacer una breve descripción sobre la necesidad de regular algunos sectores de la economía; la segunda hará un análisis del derecho comparado que incluirá el origen de la noción de agencia en los Estados Unidos y su implementación en varios países de tradición jurídica del common law, en donde tuvo su origen, y en la familia germano románica en donde también se ha implementado; en tercer lugar, se hará un análisis sobre los diferentes tipos de contratos de petróleo, la historia de la regulación del petróleo en Colombia y el origen de la ANH; en cuarto lugar se establecerá si la ANH tiene los rasgos de una agencia independiente o si, por el contrario, es una entidad descentralizada por servicios.

\section{I. ¿CON QUÉ FIN SE REGULA?}

El premio nobel de economía, Joseph E. Stiglitz, sostiene en su libro Caída libre, que uno de los motivos por los cuales se generó la crisis del 
2008 fue la ausencia de regulación por parte del Estado norteamericano en el mercado financiero. Para este economista la regulación en los mercados financieros es un tema de gran importancia por dos motivos: en primer lugar, en este mercado, al igual que en todas las actividades económicas, existe un gran interés por parte de los individuos de maximizar sus utilidades; en segundo lugar, el mercado a veces presenta faIlas que necesitan del apoyo estatal para que se puedan corregir ${ }^{1}$.

Muchos bancos se dedicaron a aprobar solicitudes de préstamos hipotecarios sin hacer un análisis detallado de la capacidad de pago del deudor. Desafortunadamente, los derivados financieros que tenían como soporte las hipotecas no fueron supervisados por las entidades reguladoras, y por tal motivo se formaron productos financieros por un valor de 100 cuando en realidad iban a terminar valiendo 70 porque su soporte dependía de un préstamo que tenía un alto riesgo de impago ${ }^{2}$.

Al igual que en los mercados financieros, otros sectores de la economía deben ser supervisados por el Estado. Sectores tan complejos como las telecomunicaciones, hidrocarburos, energía, transporte, por nombrar solo algunos, tienen una especial regulación por parte del Estado, quien se encarga de crear unos órganos, en lo posible autónomos e independientes, responsables de establecer la forma en la que estos deben operar en el mercado.

\footnotetext{
1 Stiglitz, Joseph. Caída libre. Bogotá: Taurus, 2010.

En el sistema jurídico anglosajón se utiliza el concepto public utillity para referirse a las actividades económicas que son de especial interés por parte del Estado y que, por tal motivo, deben ser reguladas. El concepto public utillity nació en el año 1877 en el caso Munn vs. Illinois. El señor Munn argumentó que su derecho a la propiedad privada se vio vulnerado por una regulación del Estado de Illinois que limitaría la actividad económica de su empresa. Ante esto, la Corte Suprema de los Estados Unidos resolvió el siguiente interrogante: ¿puede el Estado de Illinois regular la actividad económica de una empresa privada? A lo anterior respondió la Corte que los bienes privados pueden ser regulados con el fin de favorecer un interés público ${ }^{3}$.

En relación con lo anterior, el profesor español Gaspar Ariño trata de responder a la pregunta que generalmente surge con respecto a las características que debe tener el órgano encargado de regular la actividad económica de los particulares. Probablemente, una de estas características, según el doctrinante, sería la independencia del órgano regulador, la cual se predica de las empresas privadas pero también en muchas ocasiones del mismo Estado ${ }^{4}$. Esto quiere decir que los órganos reguladores deben ser imparciales en sus decisiones y libres de toda interferencia por parte del Ejecutivo y de las empresas privadas.

Al respecto, el profesor colombiano Jorge Vélez García sostiene que un buen ejemplo es el de

3 Munn vs. Illinois, 94 U.S. $113(1876)$

4 ArIÑo Ortiz, Gaspar. Principio de derecho público económico. Bogotá: Universidad Externado de Colombia, 2003, p. 767. 
las agencias independientes en Estados Unidos, las cuales se caracterizan por estar compuestas por un cuerpo colegiado de entre cinco y once personas, por lo general distribuidas entre los diferentes partidos políticos, con la finalidad de evitar que un solo partido político tome el control sobre ellas 5 .

Otro aspecto importante en los órganos reguladores es la autonomía. Para el doctrinante español Miguel Sánchez Morón, la autonomía del órgano regulador depende de la forma en la que sean designados los directivos encargados de dirigirlo, del periodo de mandato y remoción y de los requisitos de elegibilidad del director ${ }^{6}$.

En nuestro país, un buen ejemplo de autonomía e independencia es el Banco de la República. Este órgano debe tener esas dos características debido a que, por mandato constitucional, tiene el deber de regular tres puntos importantes de la política monetaria: la inflación, la tasa de cambio y la política crediticia. Por lo anterior, la Constitución de 1991, artículo 372, dotó al Banco Central de autonomía e independencia de las ramas del poder público, dejando atrás el pasado, pues hasta el año 1963 la Junta Directiva no gozaba de autonomía ni de independencia, debido a que estaba conformada por banqueros privados y era presidida por el ministro de Hacienda. De modo similar, entre los años 1963 y 1991, el Presidente de la República tuvo una gran influencia en las decisiones de la Junta Mo- netaria, por ejemplo, la tasa de minidevaluaciones diarias era decidida por el ministro de Hacienda junto con el Presidente de la República, determinación que se le comunicaba al gerente del Banco Central sin que este último tuviera posibilidad de oponerse, es decir, simplemente se limitaba a ejecutar lo que le ordenaba el Ejecutivo, de tal forma que el Banco Central dependía de la voluntad del Presidente?

El principal problema relacionado con la dependencia de los bancos centrales del Ejecutivo, según numerosos estudios lo han demostrado, es que a mayor dependencia del Banco Central con respecto a los demás órganos del poder público, habrá un aumento en la inflación. Es decir, que cuando el Banco Central es dependiente del Ejecutivo u otra rama del poder público, la regulación en la política monetaria será poco técnica, lo cual se ve reflejado en altos niveles de inflación ${ }^{8}$. De hecho, varios países han optado por dotar de autonomía e independencia a su respectivo Banco con el fin de que sea libre y autónomo en sus decisiones, que siempre terminan afectando la tasa de inflación.

Por otro lado, la producción de hidrocarburos es de gran relevancia a nivel mundial. Cada país productor se ha encargado de establecer la forma en que se regula la exploración, explotación, producción y comercialización de estos. La forma como cada país establece su órgano regulador depende de varios factores, por ejemplo, no
5 Vélez GARCIA, Jorge. Los dos sistemas del derecho administrativo. Bogotá: Institución Universitaria Sergio Arboleda, 1996, p. 206.

6 SAnchez, Miguel. Derecho administrativo. Madrid: Tecnos, 2005, p. 206.
7 Santamaría Cárdenas, Mauricio. Economía colombiana. Bogotá: Alfaomega, 2007, p. 227. Consultado en: http://www.ecb.int/ecb/orga/independence/html/index. es.html. 10 de septiembre del 2012. 
es lo mismo un ente regulador en un sistema federal que en un sistema de tradición centralista.

En Colombia, la ANH es la encargada de regular, desde el año 2003, la producción de hidrocarburos. A pesar de que en sus estatutos se establece que goza de autonomía e independencia, en la práctica el Ejecutivo tiene una gran influencia en esta, en especial en su Junta Directiva, como se verá más adelante. A continuación se expondrá la forma en que la noción de agencia se ha originado en los EE. UU. y en otros países. Vale la pena aclarar que, por lo general, la regulación de hidrocarburos varía en cada Estado.

\section{LA AGENCIA EN LOS ESTADOS UNIDOS DE AMÉRICA}

\section{A. Historia}

Las agencias han jugado un papel muy importante en el desarrollo de la economía más grande del planeta: la de EE.UU. Precisamente, la necesidad de regular diferentes sectores de su economía permitió la creación de varias agencias. La primera de ellas está fuertemente relacionada con el comercio. En los Estados Unidos, en 1887 el Congreso creó la Interstate Commerce Commission (ICC) al sancionar la Interstate Commerce Act y nació la primera agencia independiente del país del norte. Esta jugó un papel muy importante en el desarrollo económico, puesto que debía regular las tarifas del ferrocarril, un medio necesario para el intercambio comercial entre los diferentes estados, cuyas distintas líneas existentes tenían un sistema de discriminación de precios dependiendo del producto, lo que significaba que cobraban un alto precio por el transporte de algunos bienes y generaba un alza en los precios. Todo lo anterior mostró la necesidad de crear la Icc, con el fin de establecer las tarifas máximas que podían cobrar las compañías de ferrocarriles e impedir el abuso e inequidad que se estaba generando en el mercado. Desde luego, la Icc debió fijar el precio máximo basándose en criterios técnicos para evitar consecuencias en el comercio, para lo cual fue dotada de independencia del gobierno en la toma de decisiones ${ }^{9}$.

La Icc es un buen ejemplo sobre cómo las agencias han venido contribuyendo al desarrollo económico. La regulación de tarifas fue un tema tan elemental que en 1910 se le otorgó a la ıcc la facultad de regular las tarifas de la telefonía y el telégrafo. De no haberse creado esta agencia le hubiera quedado muy difícil al Congreso regular sobre cada una de estas áreas, debido a la cantidad de trabajo que el Legislativo suele tener. El hecho de contar con una agencia especializada en cada tema permite que se estudien más detalladamente los aspectos regulatorios, teniendo en cuenta el costo que implica determinada decisión y los beneficios que se pueden generar en la economía.

La economía ha sido siempre fuente de preocupación para los americanos. De hecho, entre la crisis del año 1929 y la segunda guerra mundial, Estados Unidos se vio en la necesidad de implementar varias medidas para reactivar su economía y corregir problemas sociales como el des-

Consultado en: https://www.federalregister.gov/agencies/interstatecommerce-commission, el 12 de octubre del 2012. 
empleo. Una de ellas correspondió al presidente Franklin D. Roosevelt, quien inició la creación de varias agencias como parte de la implementación del programa New Deal, el cual tuvo el respaldo del Congreso. El respaldo del legislativo fue vital para la creación de nuevas agencias puesto que este era el encargado de delegar su poder legislativo a los nuevos órganos que tendrían como fin la implementación de políticas públicas enfocadas al crecimiento económico.

Un buen ejemplo de lo anterior fue la creación de la Public Works Administration mediante la National Industrial Recovery Act, en junio de 1933. El principal objetivo de esta agencia consistió en reactivar la economía por medio de la construcción de escuelas, hospitales e infraestructura vial y así aumentar la generación de nuevos empleos debido a la mano de obra que se requirió para estas obras. Lo anterior era necesario teniendo en cuenta que la tasa de desempleo era demasiado alta en la década de los treinta, debido a la crisis de 1929. Sin embargo, a pesar de los aspectos positivos en la economía, este órgano recibió varias críticas, una de ellas consistió en la interferencia que tuvieron los demócratas y los republicanos en sus decisiones, lo cual generó retrasos en la construcción de algunas obras de infraestructura. Para evitar la intervención de los partidos políticos en las decisiones de las agencias, en 1943 se creó la Federal Works Agency, un ente independiente que tendría menos intervención por parte del Ejecutivo. Con ello se buscaba reducir las decisiones basadas en la política y aumentar las decisiones fundadas en estudios técnicos ${ }^{10}$.

10 Consultado en: http://www.presidency.ucsb.edu/ws/index. php?pid=16423, 29 de agosto de 2012.
Debido a la influencia del economista Keynes, quien proponía una mayor intervención estatal como salida a la crisis económica, entre 1930 y 1970 se creó una gran cantidad de agencias en los Estados Unidos, hecho justificado por el doctrinante Daniel E. Hall como producto del desarrollo económico que generó una gran interdependencia entre todos los sujetos que intervenían en la economía americana, tanto productores como consumidores. En consecuencia, el Estado tuvo necesidad de crear varios órganos encargados de resolver disputas, así como de controlar y vigilar la calidad de los productos. De esta manera, los consumidores se sentirían conformes con la calidad de productos como la comida, la medicina, el suministro de energía, entre otros bienes y servicios que requieren de seguridad en el proceso de producción para evitar perjuicios a los consumidores finales ${ }^{11}$.

En la actualidad, Estados Unidos necesita proveer una gran variedad de servicios como recreación, educación, asistencia para el desempleo, salud, lo cual ha ocasionado un aumento considerable en el número de personas que trabaja con el Estado: en el año 1800 solamente 3000 personas trabajaban con el gobierno (el 0.005 de la población total, que por entonces era de 5.084.000 personas) y en el año 1995 había 3.000.000 de empleados públicos, lo que representaba más del uno por ciento de la población americana ${ }^{12}$.

11 HALL, Daniel. Administrative Law Bureaucracy in a democracy. Fifth edition, Prentice Hall, 2012.

12 Hall, Daniel. Administrative Law. New Jersey: Prentice Hall 2012, pp. 15-20. 


\section{B. Marco jurídico}

Las agencias se rigen por la rama del derecho administrativo. Las funciones y procedimientos de cada una son fijadas en el momento de su creación mediante una ley elaborada por el Congreso, que se conoce con el nombre de enabling legislation; sin embargo, existe una ley general, la Administrative Procedures Act de 1946, encargada de establecer la forma en que las decisiones de todas las agencias federales pueden ser sometidas a una revisión por parte de las Cortes Federales de los Estados Unidos, lo que se conoce como Judicial review ${ }^{13}$.

Cuando el Congreso delega su poder legislativo en la agencia, esta última recibe el nombre de quasi legislative authority. El Legislativo también le puede otorgar a las agencias la capacidad de escuchar y resolver las disputas como si fueran una Corte, este poder se conoce como quasi judicial power. Por tanto, la agencia puede tener los dos poderes, es decir, tener dentro de sus funciones la quasi legislative y la quasi judicial.

La facultad del Congreso de delegar su poder legislativo fue discutida por quienes argüían que el poder del Congreso ha sido delegado por el pueblo y, por lo tanto, un órgano delegado no puede delegar su función en otros órganos. En pocas palabras, no se puede delegar lo que ya ha sido delegado. El magistrado John Marshall Harlan fue uno de los principales opositores a la tesis de delegar el poder del Legislativo.

13 Ibid
Contrario a la tesis de John Marshall Harlan, Clarke Adams sostuvo lo siguiente:

Cuando los legisladores estadounidenses se dieron cuenta de la necesidad de controlar algunos sectores de la economía llamados de interés público, y comprendieron, también, su propia incapacidad - por falta de la pericia técnica necesaria y por la continua movilidad de las actividades por controlar-, para dictar normas materiales exigidas por estos nuevos campos de intervención estatal, se vieron obligados a expedir normas instrumentales, las cuales, en lugar de establecer derechos y deberes, delegaban en otro órganos, dentro de límites bien determinados, la facultad de crear derechos y obligaciones.

La conclusión a la que llegó Clarke Adams no fue para nada fácil, puesto que hubo un fuerte enfrentamiento entre el Congreso y el poder Judicial con respecto a la facultad de delegar las funciones legislativas en las agencias. En todo caso triunfó la tesis de que el Legislativo tenía la necesidad de delegar sus funciones debido a lo que se mencionó anteriormente ${ }^{14}$.

Más tarde se estableció que en el momento de la creación de la agencia, el Congreso debe determinar unos estándares, es decir, unos límites a su facultad reguladora, al igual que unas funciones que deben estar claramente establecidas en los estatutos. Algunas sentencias han desarrollado la anterior afirmación, por ejemplo, la sentencia Unite States V. Chicago M. St. Pand

14 Consultado en: http://www.supremecourthistory.org/history-of-thecourt/associate-justices/john-marshall-harlan-1877-1911/, el 20 de octubre de 2012. 
P. R. Co de 1931 y United States V. Shreveport Grainand Elevator Co de $1932^{15}$.

Vale la pena tener en cuenta que existen potestades que son esenciales del Legislativo, es decir, que algunas facultades de este órgano no pueden ser delegadas a las agencias, por ejemplo, la ratificación de los tratados internacionales por parte del Senado.

\section{Tipo de funciones que puede tener una agencia}

Las agencias se pueden clasificar dentro de alguno de los siguientes tres tipos de funciones: el social Welfare que consiste en el apoyo que busca desarrollar el bienestar de la población vulnerada en la sociedad americana, como por ejemplo, el U.S. Department of Health and Human Resource o el U.S. Department of Veteran's Affairs; las Regulatory agencies que son las encargadas de manejar la regulación de determinado tipo de actividades industriales por medio de las licencias, es el caso de la Federal Communication Commission que determina cuáles son las empresas que pueden tener una licencia para operar en el mercado, desde luego basándose en criterios objetivos y técnicos, y la Federal Energy Regulatory Commission responsable de la regulación de las tarifas de algunos sectores como el gas y la electricidad ${ }^{16}$; por último tenemos las public service agency, que no se encargan de redistribuir el dinero como las

15 Consultado en: http://supreme.justia.com/cases/federal/us/287/77/, el 12 de octubre de 2012.

16 Hall, Daniel. Administrative Law. New Jersey: Prentice Hall, 2012, pp. 20-23. agencias de bienestar social o social wellfare o de otorgar licencias como las regulatory agency, sino de promover la investigación y suministrar información a la sociedad, por ejemplo, la National Science Foundation cuya función es realizar estudios de investigación para ofrecerle a los ciudadanos información sobre temas científicos $^{17}$.

\section{Las agencias pueden ser de dos tipos: agencias independientes y las agencias que dependen del Ejecutivo}

Las que dependen del Ejecutivo son conocidas como executive agency o agencia ejecutiva $\mathrm{y}$, por lo general, tienen las siguientes características: la elección de sus directivos la hace el Presidente de los Estados Unidos, por lo tanto cada vez que este cambia hay relevo en la dirección del órgano regulador, sin embargo, la remoción del director de la agencia se puede producir en cualquier momento; el director de la agencia debe rendirle cuentas sobre su gestión al Presidente; y, por último, se encuentran subordinadas al poder Ejecutivo. Por ejemplo, el U. S. Department of Defense tiene como cabeza al secretario de defensa el cual es designado por el Presidente de los Estados Unidos con el consentimiento del Senado. Vale la pena aclarar que en algunos casos, para elegir al máximo jefe de la agencia no se requiere la aprobación del Senado. Este tipo de agencia debe entregar un reporte anual al Ejecutivo sobre el cumplimiento de sus obligaciones.

17 Ibid. 
Las agencias independientes, a diferencia de las anteriores, están dirigidas por un órgano colegiado conformado por miembros de los partidos Republicano y Demócrata, con la finalidad de que ninguno tenga el control absoluto sobre estas; no están en la obligación legal de rendirle reportes al Ejecutivo pero sí al Congreso, aunque cada uno de los miembros del cuerpo colegiado es elegido por el Presidente de turno con la confirmación del Congreso ${ }^{18}$; por último, cuentan con la suficiente autonomía para tomar decisiones basadas en criterios técnicos. Vale anotar que este fue el tipo de agencia que pretendía imitar la ANH, puesto que en sus estatutos dice que es autónoma e independiente y su naturaleza pasa de autoridad administrativa a agencia independiente.

\section{E. Regulación en la producción de hidrocarburos}

La regulación de hidrocarburos en los EE. UU. es bastante compleja debido a que cada estado tiene una agencia para este propósito. Por ejemplo, la Colorado Oil and Gas Conservation Commission es la encargada de regular la explotación de petróleo y gas en el estado de CoIorado $^{19}$, la Railroad Commission of Texas es la responsable de regular la explotación de petróleo en el Estado de Texas -anteriormente se encargaba de regular los ferrocarriles ${ }^{20}$.

18 Hall, Daniel. Administrative Law. New Jersey: Prentice Hall, 2012, p. 8.

19 Consultado en: http://cogcc.state.co.us/, el 10 de octubre del 2012
A pesar de lo anterior, varios estados, por medio de un tratado, fundaron la Interstate Oil and Gas Compact Commission, el 16 de febrero de 1935, con la finalidad de reivindicar la autonomía de cada estado en la explotación de hidrocarburos, unificar la legislación en esta materia, así como mejorar y compartir experiencias. Los estados pueden adherirse a ella ratificando el tratado. Entre sus funciones está hacer una serie de recomendaciones encaminadas a mejorar la explotación de hidrocarburos y el manejo de los residuos tóxicos producto de la realización de dichas actividades industriales. Algunas recomendaciones sobre el manejo de las reservas de hidrocarburos han sido de gran relevancia con el fin de que la explotación de estos recursos no renovables se haga en forma sostenible ${ }^{21}$.

\section{LA AGENCIA EN CANADÁ}

\section{A. En Canadá se encuentran las agencias que dependen del Ejecutivo y las independientes}

Dentro de las agencias dependientes se encuentran, por nombrar solo algunas: Department of Canadian Heritage que depende del Minister of Canadian Heritage y el Department of Finance Canada que depende del Minister of Finance, encargado de ayudar a desarrollar las políticas sociales y económicas implementadas por el Estado, por tanto, está fuertemente influenciado por este y es a él ante quien responde, no ante el Parlamento. El Department of Finance

21 Consultado en: http://www.iogcc.state.ok.us/, el 08 de octubre del 2012. 
Canada cumplió 150 años en el 2012. Por su condición, no hay autonomía ni independencia en este tipo de agencias.

Las agencias independientes no dependen de un ministerio determinado. Por ejemplo, la Financial Consumer Agency of Canadá fue creada en el año 2001 con la finalidad de que supervisara el cumplimiento de toda la normatividad relacionada con la protección e información a que tienen derecho los usuarios de los servicios financieros. Es curioso que, a pesar de que esta agencia es considerada un órgano independiente, su commissioner o director sea elegido por el ministro de Finanzas, para un periodo de cinco años. Lo anterior se contrapone a la tradición que tienen en Estados Unidos, en donde, como vimos, las agencias independientes cuentan con un órgano colegiado integrado por miembros sugeridos por el ejecutivo pero ratificados por el Congreso. Tal vez la independencia alude a la forma en que se puede remover su director, puesto que tiene que haber un error grave para que sea relevado del cargo, lo cual demuestra que el ministro de Finanzas no cuenta con un amplio nivel de discrecionalidad para decidir al respecto. Actualmente, Ursula Menke es la commissioner de esta agencia; fue elegida en el año 2007 por el ministro de Finanzas, Jim Flaherty -quien ocupa dicho cargo desde el año 2006 y pertenece al partido Conservador-; su periodo finalizó en diciembre de 2012 pero fue reelegida por seis meses más. El partido Conservador sigue en el poder y por lo tanto el ministro de Finanzas es el mismo y debe elegir, por segunda vez, al Commissioner de la Financial Consumer Agency, lo cual quiere decir que durante dos periodos el director de la agencia es elegido por un mismo partido político. En Estados Unidos el cuerpo colegiado busca un equilibrio entre los dos partidos políticos con el fin de evitar que un solo partido tenga el poder de una agencia. ${ }^{22}$

A pesar de que el director de la Financial Consumer Agency debe ser independiente en la toma de sus decisiones, y que su único objetivo es la protección del consumidor, los doce años de historia que lleva esta agencia han demostrado que se ha visto politizada, debido al poder que tiene el ministro de Finanzas de turno en su elección. Por ejemplo, su primer director, William George Knight, era miembro del New democratic Party, partido político con una orientación ideológica distinta a la del partido Conservador. Por lo anterior, las regulaciones de esta agencia pueden variar según el partido político de quien esté a su cargo ${ }^{23}$.

Lo anterior es prueba de que la simple afirmación en los estatutos de creación de una agencia, relacionados con la "autonomía e independencia", no significa que en la realidad lo sea, porque hay varios factores que permiten mediante un estudio detallado mostrar su posible dependencia con respecto a los partidos políticos. Se sabe que por más que existan factores "técnicos" que justifiquen las actuaciones reguladoras de estos órganos, se puede presentar el caso en el que un partido político presente las decisiones como si fueran técnicas cuando en realidad pueden haber factores políticos de fondo.

22 Consultado en: http://www.fin.gc.ca/n07/07-093-eng.asp, el 21 de noviembre de 2012

23 Ibid. 


\section{B. Regulación en materia energética e hidrocarburos}

En cuanto a la explotación de hidrocarburos en Canadá, Alberta es la provincia que en el 2004 tuvo alrededor del 70 por ciento de la producción de petróleo, por lo tanto, allí se concentrará el análisis sobre la manera en la que diferentes organismos estatales intervienen en la producción de los hidrocarburos.

En Alberta hay tres órganos encargados de velar por la explotación sostenible de hidrocarburos: Energy Resources Conservation Board (ERCB), Alberta Department of Energy y Alberta Utilities Commission.

Energy Resources Conservation Board es una agencia independiente que tiene como finalidad regular la seguridad, la conservación y el uso eficiente del petróleo y del gas natural en la provincia de Alberta. Todos los proyectos que tienen como fin la explotación de hidrocarburos deben ser diseñados y construidos basándose en una serie de regulaciones técnicas dictadas y supervisadas por la ERCB24.

Alberta Department of Energy es el encargado de ejecutar todo lo relacionado con la política energética de la provincia. Lo anterior también incluye el sector de la minería ${ }^{25}$.

Alberta Utilities Commission (Auc) es la agencia responsable de proteger a los consumidores a través de la regulación de los precios de la

\footnotetext{
24 Consultado en: http://www.ercb.ca/, 18 de agosto de 2012.

25 Consultado en: http://www.energy.alberta.ca/ 20 de agosto de 2012.
}

electricidad y del gas. El gas es un hidrocarburo importante para el desarrollo industrial, por tal motivo este órgano se encarga de supervisar un suministro eficiente y tarifas razonables en el precio, por medio de la vigilancia de las empresas que lo suministran, como AltaGas Utilities, ATco Gas y Direct Energy Regulated Services, las cuales deben contar con la aprobación de sus tarifas por parte de la agencia reguladora ${ }^{26}$. La Auc también se encarga de aprobar las tarifas, así como los términos y condiciones que estas empresas imponen a sus usuarios con el fin de evitar abusos ${ }^{27}$.

Por último, la National Energy Board es una agencia federal e independiente, creada por el Parlamento en 1959, con el propósito de regular el intercambio de gas y petróleo entre las diferentes provincias ${ }^{28}$. Por ejemplo, la construcción de la infraestructura que conecta las provincias de Canadá o la que permite la conexión con Estados Unidos para el intercambio de energía, petróleo o gas.

Todo lo anterior demuestra que en Canadá hay una serie de agencias que permiten que los temas regulatorios se manejen en forma adecuada, en pocas palabras, que respondan a los requerimientos técnicos que requiere cada actividad económica o industrial.

27 Consultado en: http://www.auc.ab.ca/Pages/Default.aspx, el 12 de agosto de 2012.

Consultado en: http://www.neb-one.gc.ca/clf-nsi/rcmmn/hm-eng.html, el 10 de agosto de 2012. 
Por otro lado, el beneficio de tener agencias independientes se ve reflejado en el hecho de que si los productores no estuvieran regulados, los usuarios serían víctimas de grandes abusos. Vale la pena recordar el caso de EE. UU. antes mencionado, en relación con las tarifas altas que imponían los ferrocarriles a los agricultores.

\section{LA AGENCIA EN AUSTRALIA}

En Australia, al igual que en los Estados Unidos, se han creado varias agencias con el fin de regular distintos sectores de la economía. Allí las agencias reciben el nombre de statutory authority y pueden presentarse a nivel federal y estatal. En el primer caso, el poder Legislativo es delegado por el Parlamento Australiano en la statutory authority; en este caso, cada órgano regulador tiene su propia enabling legislation u originating act, es decir, la ley de origen 0 , en otras palabras, la que le dio vida. Mediante dicho acto también se establecen las funciones de la agencia. En el segundo caso, cuando el órgano regulador es de nivel estatal, el Queensland Parliament, es decir, la Asamblea Legislativa del Estado de Queensland le da vida por medio de una ley, con la finalidad de que tenga competencia únicamente en el estado en el que fue creado. Para aclarar lo anterior, a continuación se hará una breve explicación de las agencias que se encargan de regular la explotación de recursos y la producción de energía en Australia ${ }^{29}$.

En primer lugar, a nivel federal, la Australian Renewable Energy Agency (ARENA) es una statutory

29 Consultado en: http://australia.gov.au/topics/law-and-justice, el 12 de octubre de 2012. authority independiente que trabaja por la promoción y el desarrollo de energías renovables a través de varios programas de investigación que buscan promover la obtención de energía limpia por medio de la biomasa, la geotérmica, la hidroeléctrica, la solar, entre otras. Este órgano ha invertido alrededor de 1.7 billones de dólares australianos en varios proyectos de investigación. Para el cumplimiento de sus objetivos es importante que la aprobación y diseño de los proyectos respondan a fines totalmente técnicos, de modo que los resultados estén enfocados a obtener energía más limpia con el fin de mitigar el impacto negativo del cambio climático. La independencia de esta agencia se predica con base en que es un órgano colegiado conformado por seis miembros elegidos para un periodo inamovible, por el ministro de Recursos y Energía, con la participación del presidente del Department of Resources, Energy and Tourism. El director de esta agencia es postulado por el cuerpo colegiado y ratificado por el ministro de Recursos y Energía, con la condición de que tenga experiencia en el tema. Actualmente, dentro del cuerpo colegiado se encuentra Jane Sargison, quien ha estudiado ingeniería mecánica; Mar Twidell, exdirector del Instituto Australiano Solar y Greg Bourne, titulado en química pura. Lo anterior muestra que la agencia está conformada por especialistas en el tema, lo cual permite que las decisiones que se tomen cada día estén directamente relacionadas con la manera de incentivar la obtención e investigación de energía limpia ${ }^{30}$.

30 Consultado en: http://www.ret.gov.au/energy/clean/arena/Pages/arena.aspx, el 12 de noviembre del 2012. 
La Australian Competition and Consumer Commission (ACCC) es una statutory authority federal e independiente, que se encarga de regular todo lo relacionado con la competencia entre las diferentes empresas bajo la Competition and Consumer Act 2010, promoviendo la competencia y el libre mercado, con el fin de que los consumidores puedan obtener grandes beneficios en los precios de los bienes y servicios, debido a que se busca reducir las prácticas monopólicas que elevan los precios de los productos en el mercado ${ }^{31}$.

En materia de hidrocarburos, las regalías que se obtienen son compartidas por el Estado y la Commonwealth of Australia (Confederación de Australia), por ejemplo, el estado de Western Australia y el Gobierno. En el año 2011, el estado de Western Australia obtuvo alrededor de 955 millones de dólares australianos por las regalías generadas por la explotación de hidrocarburos $^{32}$. Con base en lo anterior se puede inferir que los hidrocarburos deben tener una regulación en su explotación y en el manejo de la gran cantidad de dinero que se obtiene por este concepto. Al igual que en Canadá y Estados Unidos, la regulación de los hidrocarburos está a cargo de cada estado, en este caso el Department of Mines and Petroleum (DMP) es una agencia en el estado de Western Australia que establece toda la regulación para la obtención de títulos mineros y permisos de explotación petrolera. El pago de las regalías por explotación de petróleo es

\footnotetext{
31 Consultado en: http://www.accc.gov.au/content/index.phtml/ite$\mathrm{mld} / 142$, el 12 de noviembre del 2012.

32

Consultado en: http://www.dmp.wa.gov.au/1943.aspx, el 13 de noviembre del 2012
}

fijado por este órgano, y oscila entre el 10 y 12 por ciento de la producción obtenida ${ }^{33}$.

La protección del medio ambiente en el estado de Western Australia está a cargo del Department of Environment and Conservation, el cual emite las licencias ambientales de conformidad con los requisitos establecidos por la regulación ambiental vigente ${ }^{34}$.

Como se ve, en Australia hay ciertas funciones que se manejan por medio de agencias federales, mientras que otros asuntos como la explotación de hidrocarburos son manejados por agencias estatales. En Colombia no hay un sistema federal como en Australia, por tal motivo la regulación para la explotación de hidrocarburos está a cargo de la ANH, agencia que regula toda la producción a nivel nacional.

\section{LA AGENCIA EN FRANCIA}

En Francia, las agencias reciben el nombre de autorité administrative indépendante (AAI). Aparecieron en 1978 cuando el Legislador creó la Commission Nationale Informatique et Libertés y se encargó de emplear dicho término para referirse a estas.

\section{A. La evolución de la autonomía en los entes estatales en Francia}

Cuando el Legislador creó la primera Aal fue una novedad, puesto que Francia tenía una tradi-

\footnotetext{
33 Consultado en: http://www.dmp.wa.gov.au/4755.aspx

34 Consultado en: http://www.dec.wa.gov.au/about-us.html
} 
ción centralista y bastante burocrática, según el doctrinante español Ramón Parada. Si bien a finales del siglo xIx y comienzos del siglo xx la descentralización por servicios surgió como respuesta a dicha tradición centralista, la autonomía e independencia de los órganos descentralizados por servicios era mínima.

El doctrinante francés Hauriou sostiene que la descentralización funcional en Francia surgió con el fin de encomendar la prestación de servicios a los establecimientos públicos, para evitar otorgarles un mayor nivel de autonomía y poder político a los municipios y departamentos $^{35}$. Sería un peligro que existiera la panadería municipal, la farmacia municipal, la carnicería municipal, entre otros establecimientos, pues el alcalde, teniendo el control sobre todos esos establecimientos podría cometer muchos abusos y más aún cuando el poder socialista local era bastante fuerte en el siglo xx. Por tal motivo no se les otorgó mayor autonomía a los municipios, optando por la creación de establecimientos públicos con un nivel mínimo de independencia ${ }^{36}$.

Sin embargo, la descentralización no implicaba independencia y autonomía absolutas por parte de los establecimientos públicos. Estos últimos fueron las primeras personas jurídicas de origen estatal distintas a los municipios, departamentos y la nación. En el caso colombiano, la ANH ha sido constituida como persona jurídica, sin embargo, esto no garantiza su independencia y autonomía, simplemente se puede considerar un

35 Consultado en: http://www.hauriou.net/, el 10 de octubre de 2012

36 PARAdA, Ramón. Derecho administrativo II. Madrid: Marcial Pons, 2008, p. 187. paso hacia estos dos conceptos. La ANH, al igual que los establecimientos públicos, pertenece al sector descentralizado por servicios, que mantiene un nivel mínimo de autonomía, pero esta no es la misma que se predica en la agencia del sistema norteamericano, donde es mucho más compleja.

La creación de una nueva categoría de ente público, en este caso las administraciones independientes, en Francia permitió que el nombramiento de los titulares de sus órganos directivos no lo hiciera el gobierno sino el Parlamento, Corte de Casación, entre otros, lo que garantiza su independencia ${ }^{37}$. Declarar inamovibles a los directivos durante un periodo determinado también es una garantía de independencia, debido a que podrán realizar su gestión sin temor a ser removidos de su cargo por el gobierno. En este punto la independencia se asimila a la agencia independiente de los Estados Unidos.

\section{LA AGENCIA EN ESPAÑA}

En España surgió la necesidad de crear las administraciones independientes de regulación y supervisión por los siguientes motivos, según el doctrinante Gabriel Fernández Rojas:

1. En los años anteriores a la creación de las agencias hubo una gran manipulación partidista de las administraciones públicas.

2. El perfeccionamiento de los mecanismos institucionales para la protección de la salud pública, la preservación de un medio ambiente

37 Idem 
sano, y la garantía de los derechos y libertades fundamentales.

3. La defensa de un mercado eficiente y con alta calidad e incertidumbre competencial, para sostener y potenciar un elevado nivel de desarrollo.

4. El incremento de la competitividad, la eficiencia y la calidad de las administraciones públicas en el marco de la Unión Europea ${ }^{38}$.

Lo anterior muestra que, sin importar el nombre que tengan, las agencias, en el caso español se conocen como administraciones independientes de regulación y supervisión. Estas deben cumplir con determinados objetivos que requieren de la no intervención por parte de los partidos políticos, con la finalidad de que las decisiones se basen en criterios técnicos y autónomos. Esto ha permitido que su creación brinde transparencia, imparcialidad y neutralidad en la administración pública. De la afirmación que las administraciones públicas son independientes se podría inferir en forma ligera que gozan de dicha calidad en un ciento por ciento, sin embargo hay que reconocer que estos órganos deben trabajar junto con el Ejecutivo, con el fin de que las decisiones sean realmente implementadas en el territorio español, lo cual no afecta en forma significativa su independencia.

Por otro lado, es necesario tener en cuenta el origen y las facultades que se les otorgan, puesto que si bien son independientes, deben acatar los principios constitucionales. El Parlamento les ha otorgado la facultad de supervisar, es de-

38

RoJas FERnÁndez, Gabriel. Derecho administrativo, 2005 cir, la potestad para autorizar, vigilar y sancionar, pero con limitación a que regulen exclusivamente los sectores económicos que se les han asignado previamente. Esto da garantía de que en cada sector vigilado por determinada administración independiente, los administrados gocen de una imparcialidad derivada del conocimiento técnico. De nada serviría crear agencias que no están sometidas a unos parámetros previamente establecidos por el Parlamento ${ }^{39}$.

Para definir los parámetros que garanticen la imparcialidad de las administraciones independientes, el Parlamento sanciona una ley en la cual establece en forma clara sus facultades. Por ejemplo, la Ley 12 de 1997, sobre la liberalización de las telecomunicaciones, le otorgó a la Comisión del Mercado de las Telecomunicaciones la facultad de regular este campo. Posteriormente, el legislador se dio cuenta de que en el momento de iniciar la liberalización de las telecomunicaciones comenzaron a funcionar nuevos operadores y era de esperarse que entre estos surgieran conflictos, por lo que asignó a la Comisión la competencia arbitral, por medio de la Ley 32 de 2003, reconociendo, entre otras capacidades, su amplio conocimiento en el tema. Lo anterior muestra que el legislador, cuando lo considere necesario, puede aumentar las funciones de los órganos independientes, de lo contrario deben seguir con los parámetros que se les han asignado en el momento de su constitución. La asignación de las funciones de estos órganos, por ley, permite que los administrados tengan conocimiento sobre sus procedimientos y regulaciones, entre otros temas que deben ser

$39 \quad$ Ibid 
establecidos en forma previa para evitar cualquier tipo de arbitrariedad.

El Parlamento también ha creado administraciones públicas que contemplan dentro de sus estatutos la facultad de vigilancia. Por ejemplo, la Comisión del Mercado de las Telecomunicaciones también tiene la función de vigilar a los operadores, de tal forma que puede abrir investigaciones y acceder a documentación relevante, comprobar y examinar la legalidad y veracidad de los soportes, citar y tomar declaraciones e inspeccionar las sedes de los operadores sectoriales.

Por último, las administraciones independientes tienen la función de sancionar. Esta función también se le ha atribuido a la Comisión del Mercado de las Telecomunicaciones en el marco de la potestad arbitral sobre los conflictos que puedan surgir entre los operadores de redes y servicios. Las partes involucradas en el conflicto pueden someterse a través de un convenio previsto por la ley de arbitraje, pero faltando este acuerdo de sumisión la potestad arbitral y la decisión que se dicte es plenamente pública y contra ella no habrá otro recurso más que el contencioso administrativo.

En conclusión, las administraciones han tenido tres grandes funciones: primera la de autorizar, segunda la de investigar y, por último, la de sancionar. El Parlamento es el encargado de asignarle dichas funciones a las administraciones con el fin de que estas se muevan dentro de los parámetros establecidos por la ley ${ }^{40}$.

40 PARADA, Ramón. Derecho administrativo II. Madrid: Marcial Pons, 2008.

\section{A. La elección de los directivos en las Autoridades Administrativas Independientes (AAI)}

La forma de elegir al director de una AAl depende de cada administración, por ejemplo, en la Agencia Española de Protección de Datos su director es nombrado por el Gobierno, de entre uno de los miembro del Consejo Consultivo, el cual está compuesto por un diputado y un senador, tres representantes de las administraciones públicas, un representante de los titulares de ficheros privados, uno de la organización de consumidores y usuarios, un miembro de la Real Academia de la Historia y un experto en la materia propuesto por el Consejo de Coordinación Universitaria. Lo más importante es que el director no recibe ninguna instrucción por parte del Gobierno a pesar de que este haya participado en su elección; tampoco puede ser removido durante su mandato de cuatro años, a menos que incumpla gravemente sus obligaciones 0 tenga incapacidad sobrevenida para el ejercicio de su función, incompatibilidad o condena por delito doloso ${ }^{41}$.

Otro ejemplo es la Comisión del Mercado de las Telecomunicaciones, la cual se rige por un Consejo compuesto por un presidente, un vicepresidente y siete consejeros nombrados por el Gobierno mediante Real decreto, luego de un procedimiento bastante complejo: el ministro de Fomento se dirige a la Comisión competente en el Congreso de Diputados con el fin de presentar a una persona que sea de reconocida idoneidad profesional en el sector de las tele-

41 Ibid 
comunicaciones y regulación de mercados; una vez hecho lo anterior, el Ministerio lo discute con un secretario designado por el Consejo, quien a pesar de tener voz pero no voto puede intervenir en la elección del futuro miembro; por último, el ministro de Fomento le propone al Gobierno el futuro Consejero para que este apruebe su elección por medio de un Real Decreto.

Si comparamos el sistema Español con el de los Estados Unidos es fácil ver que hay algunas diferencias. En todo caso, en materia de autonomía e independencia, España trata de seguir el modelo americano estableciendo en las AAl varias características comunes a la agencia independiente: un órgano colegiado, directivos con experiencia en el tema regulatorio y elección durante un determinado periodo, en el cual son inamovibles a menos que cometan una falta grave.

\section{LA AGENCIA EN SUECIA}

En Suecia, cada agencia depende y está sujeta a un determinado Ministerio con el que en forma conjunta debe desarrollar los objetivos que tengan en común. A pesar de lo anterior, es curioso que ni el Ministerio ni el ministro tengan el control sobre las agencias, puesto que siguen gozando de autonomía e independencia, por lo tanto, el día a día de lo que hace cada una de ellas no depende de lo que diga el Ministerio. Esta prohibición se encuentra explícita dentro de la legislación sueca. Por ejemplo, la Swedish Energy Agency tiene como misión asegurar el abastecimiento de energía en forma eficiente y sin abuso en los precios para el consumidor, ve- lando por el cumplimiento de toda la regulación que hay en materia energética. Para ello goza de especial autonomía en sus decisiones, a pesar de que forma parte del Ministry of Industry, Employment and Comunications, el cual tiene alrededor de veintisiete agencias a su cargo. Sería imposible para el Ministerio supervisar y controlar todo lo que hace cada una de estas agencias, por lo tanto pertenecer a una de ellas significa hacer parte de un plan de desarrollo y colaboración pero no implica subordinación ${ }^{42}$.

La Swedish Energy Agency tiene el deber de desarrollar nuevas fuentes de energía renovable como la eólica, y en la ejecución de sus deberes cuenta con un amplio margen de discrecionalidad. Sin embargo, está obligada a publicar los resultados de su gestión, lo cual permite que los ciudadanos también la supervisen. Vale la pena destacar que aunque actualmente la producción de energía eólica no supera el cuatro por ciento de la producción total de energía, la agencia ha logrado duplicar la producción gracias a su gestión ${ }^{43}$.

Esta forma de manejar el modelo de agencia es bastante curioso, puesto que lo normal es que la agencia independiente no dependa de ningún Ministerio, sin embargo, en Suecia pertenecer a uno de ellos es una simple formalidad.

42 Consultado en: http://www.socialstyrelsen.se/, el 21 de noviembre del 2012.

43 Consultado en: http://www.energimyndigheten.se/en/About-us/Mission/Promote-the-development-of-windpower/. 
VIII. LA AGENCIA EN EL REINO UNIDO

En el Reino Unido las agencias, a diferencia de las de Suecia, son dependientes, es decir, cada una pertenece a un Ministerio determinado, al que deben responder de todas las actividades que realicen en el ejercicio de sus funciones.

Por otro lado, los quasi autonomous governmental bodies o quangos no hacen parte de ningún departamento del Estado y, por lo tanto, son responsables de sus actos ante el Parlamento británico. Un quango es el equivalente a la Independent Agency de los Estados Unidos de América.

Los quango cumplen con funciones ejecutivas, regulatorias y consultivas, dependiendo de la actividad que se les haya encargado en el momento de su constitución y son creados por medio de una ley del Parlamento británico. Por ejemplo, la Competition Commission fue creada por medio de la Competition Act de 1998; en dicha ley se le asignan o delegan ciertas facultades al quango, que con el paso del tiempo pueden ser ampliadas o recortadas por medio de una nueva ley, como sucedió cuando el Parlamento amplió las facultades de la Competition Commission con la Enterprise Act 2002 ${ }^{44}$.

\section{A. Regulación en la explotación de hidrocarburos}

La explotación de hidrocarburos en el Reino Unido tiene una gran relevancia, debido a la pre-

44 LeYLAnd, Peter. Administrative law. Oxford, 2007. sencia abundante de este recurso en el Mar del Norte, por lo tanto, es posible hacer un análisis sobre cómo se realiza la explotación de este recurso no renovable.

En Colombia la ANH tiene la tarea de hacer seguimiento al cumplimiento de las normas de seguridad en la explotación y exploración de hidrocarburos. Este tipo de actividad es realizada en el Reino Unido, con excepción de Irlanda del Norte, por la Health and Safety Executive (HSE) un quango que se encarga de estimular, regular y ejecutar todo lo relacionado con la seguridad, la salud de los trabajadores y su bienestar en el sitio de trabajo. Para lograr dicho objetivo la HSE debe velar por el cumplimiento de las normas técnicas vigentes.

Por otro lado, el Department of Energy and Climate Change es un órgano dependiente del gobierno, creado en el año 2008, con la finalidad de otorgar las licencias, exploraciones y regulación del gas y petróleo en el Reino Unido. A pesar de que no es un quango, es decir, que no goza de autonomía e independencia del Ejecutivo, su regulación está basada en fundamentos técnicos. El Department of Energy and Climate Change maneja seis tipos de licencia para la producción del petróleo que requieren, por parte del solicitante que tenga la capacidad técnica y ambiental como también el suficiente capital financiero para desarrollar el proyecto de exploración y explotación de petróleo ${ }^{45}$.

Por último, la Environment Agency es un quango que se encarga de regular todo lo relacionado

45 Consultado en: http://www.decc.gov.uk/, el 22 de octubre del 2012. 
con la protección del medio ambiente, lo cual incluye la actividad de explotación de hidrocarburos puesto que el desarrollo de dicha actividad se relaciona directamente con la contaminación ${ }^{46}$. En Colombia, la ANH también tiene esa función, según lo estipulado en la Resolución 012 de la $\mathrm{ANH}^{47}$.

Todo lo anterior muestra que en el Reino Unido la explotación de hidrocarburos es bastante compleja debido a que se requiere la intervención de varios órganos: un quango que se encarga de examinar el tema ambiental y otro quango que se encarga de verificar el cumplimiento de las normas técnicas de explotación y seguridad industrial; ambos son entes autónomos que pueden ejecutar su trabajo basándose en criterios técnicos y científicos. En Colombia existe un solo órgano que tiene muchas funciones. Por tanto se puede inferir que cada Estado tiene una forma distinta de organizar la regulación de determinado sector económico ${ }^{48}$.

Consultado en: http://www.environment-agency.gov.uk/, el 23 de octubre del 2012. Consultado en: http://www.anh.gov.co/es/index.php?id=74, el 22 de octubre del 2012.

LeYLANd, Peter. Administrative law, Oxford, 2007.
IX. LA AGENCIA EN COLOMBIA: ORIGEN Y DESARROLLO

\section{A. Los tipos de contratos de hidrocarburos como forma de regular la producción de este recurso no renovable}

La inversión extranjera para la explotación de hidrocarburos es de gran relevancia y depende, principalmente, de dos factores. El primero, y tal vez el más importante, son las condiciones legales que permiten la explotación de este recurso, debido a que la empresa petrolera busca que su inversión se vea retribuida. El segundo, la estabilidad del marco regulatorio, es decir, que el Estado no lo esté cambiando. Probablemente lo anterior se podría solucionar de dos formas: teniendo un ente regulador independiente para que las decisiones regulatorias no dependan de las políticas del gobierno de turno, y, por otro, suscribiendo tratados de protección a la inversión extranjera que brinden seguridad a los inversionistas foráneos.

El profesor Wälde sostiene que la forma de contratar en cada Estado depende del ciclo político del país receptor de la inversión extranjera. Primero, el Estado puede tener reservas de petróleo y su explotación verse limitada por una regulación excesiva e inestable, junto con condiciones de inestabilidad política que pueden resultar adversas a los intereses de los inversionistas extranjeros. Por lo tanto, la implementación del contrato de concesión es utilizada para atraer al inversionista extranjero debido a que la empresa petrolera obtendría altas utilidades de- 
rivadas de la concesión. Por otro lado, el Estado que tiene una gran inestabilidad política debe renunciar a su derecho jurisdiccional porque la empresa petrolera no confiará en que las instancias judiciales fallen con base en la imparcialidad, por lo tanto, el Estado firma acuerdos de arbitraje internacional en donde se compromete a que todas las disputas que puedan surgir entre la empresa petrolera y el Estado serán dirimidas en un tribunal arbitral ${ }^{49}$.

Con base en lo anterior, se procederá a explicar el contrato de concesión y otro tipo de contratos que han sido de gran relevancia para la explotación de hidrocarburos en el mundo.

La concesión tradicional en Estados Unidos es similar a la que se usa en la explotación minera. En 1850 se inició la explotación industrial del petróleo en el país del norte y con ello surgió el contrato de concesión. Este contrato se caracterizaba por lo siguiente: el territorio que abarcaba la concesión era muy extenso; la duración era bastante larga, por lo general 60 años -en Kuwait llegó a pactarse una concesión por noventa años-; el concesionario era el propietario de todo el petróleo que se obtenía en la explotación; en algunos casos el país que otorgaba la concesión firmaba cláusulas con la empresa petrolera con el fin de eximirla de aduana, entre otros tributos. Este tipo de contratos, tan a favor de las empresas petroleras, en muchos casos eran suscritos por países en vía de desarrollo con el fin atraer la inversión extranjera.

49 Moreno, Ferney. Temas de derecho Petrolero. Bogotá: Universidad Externado de Colombia, 2010, pp. 29-45.
Después de la segunda guerra mundial se empezó a realizar un nuevo tipo de contrato llamado Product Sharing Agreement o acuerdo por asociación, que consistía en que la empresa petrolera era un simple contratista, es decir, ya no era un concesionario al que le pertenecía todo el petróleo que obtenía en la explotación, y como tal se hacía acreedora de una serie de derechos patrimoniales de compensación de costos y de remuneración. En este tipo de contrato la empresa petrolera tiene una serie de tareas y de compromisos, como por ejemplo realizar todas las actividades de exploración por su cuenta y conseguir los equipos necesarios para la explotación de petróleo, a cambio de una contraprestación que el Estado fija con la empresa petrolera. Este tipo de contrato nació debido a tres factores: primero, el aumento en la demanda de petróleo; segundo, el descubrimiento de nuevos pozos de petróleo; y tercero, la entrada de nuevas empresas petroleras que competían entre sí. Es decir, ya no existían países que competían por atraer inversionistas extranjeros sino que, por el contrario, las empresas petroleras peleaban entre ellas por obtener un contrato de Product Sharing Agreement con el país petrolero.

Tiempo después surgió un nuevo tipo de contrato conocido como Risk Service Agreement (RSA), consistente en que la empresa petrolera actuaba como mero prestador de servicios porque el Estado era el único propietario del petróleo que se obtenía en la explotación. Este resultó poco atractivo para las empresas petroleras debido a su baja utilidad.

Más adelante apareció el contrato de exploración y producción (E \& P), que se caracteriza por 
ser lo que a nivel internacional se conoce como un contrato de concesión moderna. Está basado en impuestos y regalías que debe pagar la empresa petrolera de acuerdo con la cantidad de petróleo que obtenga.

El contrato E \& P es el que actualmente maneja la ANH en Colombia. Está regulado por el Acuerdo 008 de 2008, que fue aprobado por el Consejo Directivo de la ANH. Este Acuerdo reúne temas sumamente importantes, puesto que establece las condiciones en las que se deben asignar las áreas para la explotación y exploración de hidrocarburos, especialmente los aspectos técnicos. En el caso colombiano se empezó implementando el contrato de concesión, luego se cambió al de asociación y hoy en día se aplica el contrato de exploración y explotación.

Un estudio realizado por Johnston encuentra que, en 2001, el 44\% de los contratos a nivel global son del tipo concesional, el $48 \%$ son de asociación bajo el esquema de producción compartida y el $8 \%$ son contratos de servicios. En Colombia se ha optado por un contrato de tipo concesional ${ }^{50}$.

\section{B. Historia de la regulación de hidrocarburos}

La historia del petróleo en Colombia se remonta a antes de la conquista española. Cuando Gonzalo Jiménez de Quesada llegó con sus tropas a lo que hoy es Barrancabermeja, encontró que los indígenas Yaríguíes usaban un aceite negro,

50 Consultado en: http://www.ecopetrol.com.co/, el 23 de septiembre de 2012. el petróleo, como reconfortante corporal. Más tarde, los españoles lo usaron para impermeabilizar las embarcaciones. En ese momento el petróleo tenía usos distintos a los que tiene hoy en día 51 .

Fue hasta el año 1904 cuando José Joaquín Bohórquez obtuvo, por accidente, en el Magdalena Medio, las primeras muestras de petróleo en Colombia que fueron usadas con fines industriales $^{52}$. Debió pasar solo un año para que el Gobierno Nacional firmara dos contratos de concesión: el primero, en la misma zona en la que el señor José Bohórquez obtuvo las primeras muestras del hidrocarburo; el segundo, conocido como Concesión Barco, se firmó en 1905 y tuvo una gran relevancia para el país, puesto que fue un paso importante para la construcción de la primera refinería cerca de la ciudad de Cúcuta ${ }^{53}$. En aquella época no había una regulación clara para otorgar las concesiones, no se evaluaba en forma detallada la capacidad financiera del contratista, la firma del contrato no se basaba en criterios técnicos sino en la discrecionalidad del Gobierno, por lo cual el contrato de concesión era bastante amplio.

Ecopetrol nació con la Ley 165 de $1948^{54}$ y desde ese momento una serie de concesiones petroleras comenzaron a ser administra-

51 Consultado en: http://www.ecopetrol.com.co/, el 24 de septiembre del 2012

52 Consultado en: http://www.ecopetrol.com.co/contenido.aspx?cat/D=1 49\&conID=255\&pag $\mid \mathrm{D}=928$, el 21 de octubre del 2012

53 Consultado en: http://www.ecopetrol.com.co/especiales/carta_petrolera0206/pag24.htm, el 22 de octubre del 2012.

54 Consultado en: http://www.ecopetrol.com.co/especiales/Libro60anios/ esp/cap2-5.htm, el 18 de octubre del 2012. 
das por la nueva empresa colombiana, con la característica de ser lo que se conoció a nivel mundial como las National Oil Companies (NOC), un tipo de empresas que surgió después de la segunda guerra mundial, porque muchos países estaban en desacuerdo con las compañías internacionales de petróleo, en especial por el desprestigio que tuvo el contrato de concesión que estas defendían fuertemente. Cada Estado comenzó a crear su propia empresa con el fin de que esta se encargara de explorar, explotar, producir y comercializar los recursos no renovables de su país como lo son los hidrocarburos. Algunas de estas empresas fueron: en Brasil, Petrobras, en1953; YPF en Argentina, en 1958; AGIP, en Italia, en 1926; Dermina, en Indonesia, en 1947; entre otras.

La creación por parte del Estado de las compañías petroleras obedece también a la idea del economista John Maynard Keynes, quien influyó fuertemente en las ideas del pensamiento económico al sostener que en época de crisis, como la de 1929, el Estado debe aumentar su intervencionismo y regulación en la economía, al igual que en el gasto público con el fin de que esto se vea reflejado en un crecimiento del consumo. Muchos Estados, para salir de la crisis surgida en la posguerra, comenzaron a aumentar su intervencionismo y regulación en la economía. Las noc fueron producto de dicho intervencionismo estatal: el Estado se apropia de los recursos no renovables y evita que las compañías extranjeras obtengan el control, por medio de contratos de concesión sobre un sector tan importante para el crecimiento económico.
En la década de 1970 Colombia tuvo una producción de petróleo entre 150.000 y 200.000 barriles por día (bpd) y entre 1974 y el año 2003 implementó el contrato de asociación con el fin de atraer inversionistas extranjeros. En esa época, Ecopetrol era la empresa encargada de regular el contrato de asociación, al tiempo competía con las demás empresas petroleras.

\section{El concepto de agencia es nuevo en Colombia}

El sector descentralizado por servicios está compuesto por: los establecimientos públicos, las empresas industriales y comerciales del Estado, las superintendencias con personería jurídica, las Unidades Administrativas Especiales, las empresas sociales del Estado, las empresas oficiales de servicios públicos domiciliarios, los institutos científicos y tecnológicos, las sociedades públicas, las sociedades de economía mixta y las demás entidades que cree, organice o autorice la ley para que formen parte de la Rama Ejecutiva del poder público ${ }^{55}$.

En el año 2003 Ecopetrol pasó a llamarse Ecopetrol S. A. transformándose en una sociedad pública por acciones, con tres órganos de dirección: la Junta Directiva, la Asamblea General de Accionistas y el Presidente de la compañía. Ese mismo año nació la ANH como una Unidad Administrativa Especial aunque siempre ha Ilevado el nombre de agencia. Sin embargo, en el año 2011 pasó a convertirse en agencia estatal, de tal forma que cambió su naturaleza jurídica. En

55 Vidal Perdomo, Jaime. Derecho administrativo. Bogotá: Legis, 2004, pp. 104-112. 
Colombia se empezó a hablar de agencia como si esta fuese la naturaleza jurídica del órgano regulador. El Decreto 4137 de 2011 establece lo siguiente: "Cámbiese la naturaleza jurídica de la Agencia Nacional de Hidrocarburos -ANH-, de Unidad Administrativa Especial con personería jurídica, patrimonio propio, autonomía administrativa y financiera a la de Agencia Estatal, del sector descentralizado de la Rama Ejecutiva del Orden Nacional, con personería jurídica, patrimonio propio y autonomía administrativa, técnica y financiera, adscrita al Ministerio de Minas y Energía"56.

La ANH está fuertemente influenciada por el Ejecutivo, debido a que el Presidente de la República puede intervenir en la toma de decisiones, aun cuando se supone que es "autónoma e independiente". A continuación se hará una descripción sobre la forma en que la ANH ejerce sus funciones y en que sus directivos son elegidos.

El artículo 6 del Decreto 4137 de 2011 establece que la administración y dirección de la ANH depende del Director y del Consejo Directivo. Estos dos se encargan de tomar las decisiones más relevantes relacionadas con la regulación de hidrocarburos. Dentro de las principales funciones que tiene la ANH se encuentran las siguientes: diseñar, negociar y establecer las condiciones del contrato de exploración y explotación de hidrocarburos en el país; analizar el potencial hidrocarburífero del país; asignar las áreas de exploración y explotación en el país; dirigir y coordinar la liquidación de regalías, en-

56 Consultado en: http://www.anh.gov.co/es/index.php?id=66, el 14 de octubre del 2012. tre otros. Todas esas funciones demuestran que los órganos administrativos de la ANH tienen una gran responsabilidad, debido a que están manejando toda la producción de hidrocarburos del territorio colombiano. En el estudio comparado se vio que algunos países, como Reino Unido, han optado por asignar todas las funciones que en Colombia tiene la ANH a varios órganos reguladores, es decir, no las concentran en uno solo. Países con un sistema federal, como Canadá y Australia, prefieren que cada región administre y regule sus recursos no renovables y se encargue de compartir las regalías obtenidas con los demás estados de la unión.

En cuanto a la elección del director de la ANH, el Presidente de la República es el encargado de elegirlo y removerlo, es decir, que a diferencia de otros países que se estudiaron anteriormente, en Colombia el director no se elige por un periodo determinado sino que el Presidente puede elegirlo y removerlo cuando quiera. En las agencias independientes, por ejemplo, en Canadá, la Financial Consumer Agency tiene a su director durante un periodo de cinco años en los que es inamovible.

El Consejo Directivo está conformado por los siguientes miembros: el ministro de Minas y Energía quien lo preside, el ministro de Hacienda y Crédito Público, el director del Departamento Nacional de Planeación, el director del Servicio Geológico Colombiano, el director de la Unidad de Planeación Minero Energética y dos representante del Presidente de la República ${ }^{57}$. Esta

57 Consultado en: http://www.anh.gov.co/es/index.php?id=66, el 14 de octubre del 2012. 
composición demuestra que las decisiones del Consejo Directivo también dependen de la voluntad del Ejecutivo.

\section{CONCLUSIÓN}

El análisis comparado hizo posible comprender dos cosas importantes: la primera, que el concepto de agencia independiente puede tener un nombre distinto en cada ordenamiento jurídico (quango, Autorité Administrative Indépendante, statutory authority) sin que este influya en la naturaleza del órgano regulador, lo que importa es la forma en que sus directivos son elegidos y removidos. En el caso colombiano, la Agencia Nacional de Hidrocarburos (ANH) establece en sus estatutos, que ya no es una Unidad Administrativa Especial sino una agencia estatal que goza de autonomía e independencia. Sin embargo, si bien la ANH es un ente descentralizado, con personería jurídica, lo cual le permite contratar libremente, esto no la convierte en una agencia. Por el contrario, aún sigue perteneciendo al sector de servicios descentralizados. Si se tiene en cuenta el análisis que se hizo de la Autorité Administrative Indépendante, en Francia, es posible concluir que la ANH se encuentra en un punto medio, de un proceso evolutivo que conduce hacia la independencia y la autonomía. A comienzos del siglo xx Francia comenzó a crear establecimientos públicos con el fin de dotar de autonomía a los respectivos entes administrativos. Sin embargo, a medida que fue pasando el tiempo, debió optar por entes con mayor autonomía y fue en ese momento cuando nacieron las Aal. La ANH sigue perteneciendo al sector de entidades descentralizadas por servicios que tienen autonomía, pero no en el sentido de la agencia independiente de los Estados Unidos.

En segundo lugar, en la ANH el Ejecutivo tiene el control sobre la contratación de hidrocarburos desde 1905, fecha en que se firmó la primera concesión, hasta la actualidad; opera simplemente como un órgano que facilita el trabajo del Ministerio de Minas y Energía; no es una agencia que haya recibido poderes delegados del Congreso, y mucho menos fue creada por una ley sino por medio de un decreto del Presidente de la República que fijó los estatutos y determinó que la Junta Directiva y su director dependan del Presidente de turno. Por lo anterior se puede afirmar que la ANH no goza de la independencia que caracteriza a la agencia en otros países.

En síntesis, en Colombia la ANH da la impresión de ser una agencia como cualquier otra en el derecho comparado, cuando en realidad es una entidad que tiene personería jurídica y cumple con la función de colaborarle al Ministerio de Minas y Energía en su trabajo. Además, cada decisión de la ANH depende de la voluntad del Ejecutivo. Algunos doctrinantes proponen que la regulación es buena cuando tiene independencia y autonomía en sus decisiones, sin embargo, es posible que requiera de tiempo. Por ejemplo, el Banco de la República es un órgano que dependía del Ejecutivo, pero que con el paso de Ios años adquirió autonomía en sus decisiones.

\section{Bibliografía}

Parada, Ramón. Derecho administrativo II. Madrid: Marcial Pons, 2008. 
Moreno, Ferney. Temas de derecho petrolero. Bogotá: Universidad Externado de Colombia, 2010.

Stiglitz, Joseph. Caída libre. Bogotá: Taurus, 2010.

Ariño Ortiz, Gaspar. Principio de derecho público económico. Bogotá: Universidad Externado de Colombia, 2003.

VÉlez GARCíA, Jorge. Los dos sistemas del derecho administrativo. Bogotá: Institución Universitaria Sergio Arboleda, 1996.

SÁnchez, Miguel. Derecho administrativo. Madrid: Tecnos, 2005.

Santamaría Cárdenas, Mauricio. Economía colombiana. Bogotá: Alfaomega, 2007.

Hall, Daniel. Administrative Law Bureaucracy in a democracy. Fifth edition, Prentice Hall, 2012.

Vidal Perdomo, Jaime. Derecho administrativo. Bogotá: Legis.

\section{Legislación}

Decreto 4137 de 2011.

\section{Jurisprudencia}

Munn vs. Illinois, 94 U.S. 113 (1876).

\section{Webgrafía}

http://www.ecb.int/ecb/orga/independence/ html/index.es.html. http://www.presidency.ucsb.edu/ws/index.

http://observatoriopetrolero.blogspot. php?pid=16423.

http://cogcc.state.co.us/

http://www.iogcc.state.ok.us/

http://www.ercb.ca/

http://www.energy.alberta.ca/

http://www.auc.ab.ca/Pages/Default.aspx

http://www.neb-one.gc.ca/clf-nsi/rcmmn/hmeng.html.

http://www.ret.gov.au/energy/clean/arena/Pages/arena.aspx.

http://www.accc.gov.au/content/index.phtml/ itemld/142.

http://www.dmp.wa.gov.au/1943.aspx.

http://www.dmp.wa.gov.au/4755.aspx

http://www.dec.wa.gov.au/about-us.html

http://www.socialstyrelsen.se/

http://www.energimyndigheten.se/en/Aboutus/Mission/Promote-the-development-of-windpower/.

http://www.decc.gov.uk/

http://www.environment-agency.gov.uk/ 
http://www.anh.gov.co/es/index.php?id=74

http://www.decc.gov.uk/

http://www.environment-agency.gov.uk/

http://www.anh.gov.co/es/index.php?id=74

http://www.anh.gov.co/es/index.php?id=66 com/2010/02/la-propiedad-de-las-minas-enlos.html

http://www.glo.texas.gov/GLO/agency-administration/index.html

http://www.mnr.gov.on.ca/en/Business/ OMLC/2ColumnSubPage/STEL02_164590. html 